\title{
Neumoquiste Vertebral en Columna Lumbar: Reporte de un Caso y Revisión de la Literatura
}

\section{Vertebral Body Pneumatocyst in Lumbar Spine: A Case Report and Review of the Literature}

\author{
José Fleiderman ${ }^{1}$ Pablo Justiniano ${ }^{2}$ Ignacio Cirillo ${ }^{3}$ \\ ${ }^{1}$ Traumatólogo Jefe Equipo de Columna Hospital del Trabajador de \\ Santiago - Traumatólogo del equipo de columna Clínica Universidad \\ de Los Andes, Santiago, Chile \\ 2 Residente de Ortopedia y Traumatología Universidad de Los Andes, \\ Santiago, Chile \\ ${ }^{3}$ Traumatólogo Fellowship de Columna AOSpine, Hospital del \\ Trabajador de Santiago, Santiago, Providencia, Región \\ Metropolitana, Chile \\ 4 Residente de Radiología, Universidad de Los Andes, Santiago, Chile \\ ${ }^{5}$ Neuroradiólogo Clínica, Universidad de Los Andes, Santiago, Chile
}

Ana Maria Wilkens ${ }^{4}$ Francisco Chiang ${ }^{5}$
Address for correspondence Pablo Justiniano González, Av. La Escuela 926, Santiago, Chile (e-mail: pa.justiniano@gmail.com).

Rev Chil Ortop Traumatol 2017;58:66-70.

\section{Resumen \\ Los neumoquistes intraóseos, son lesiones cavitadas de contenido gaseoso, las cuales generalmente son encontradas como hallazgos incidentales en exámenes image- nológicos a nivel sacroiliaco, pubis, sacro, clavícula, cabeza humeral, columna cervical, torácica y en menor medida, en la columna lumbar. \\ La tomografía computarizada es el examen de elección para realizar la confirmación diagnóstica y descartar diagnósticos diferenciales, y su patrón característico se describe como una lesión de aspecto quística con atenuación por gas intralesión con rangos entre -950 y $-580 \mathrm{H}$. \\ El diagnóstico diferencial debe incluir otras posibles causas de gas intraóseo como osteomielitis, osteonecrosis, quistes óseos o subcondrales, entre otros. \\ Si bien la progresión natural del neumoquiste intraoseo es desconocida, distintos autores han descrito que en seguimientos radiológicos hasta 4 años, el tamaño de las lesiones se mantiene estable, e incluso en otros casos se han resuelto de manera espontánea, sin embargo, en otros se ha reportado un crecimiento progresivo en seguimiento de 16 meses. \\ Palabras Clave \\ - neumoquiste vertebral \\ - lumbar \\ - reporte de caso \\ En cuanto al manejo del neumoquiste intraóseo, en la mayoría de los casos reportados se ha observado una evolución benigna, con manejo conservador de las lesiones, sin embargo frente a lesiones de gran tamaño con riesgo de fractura patológica se debe plantear la posibilidad de manejo quirúrgico con corpectomía e injerto óseo. \\ Debido a la escasa evidencia respecto de esa lesión, es que se reporta un caso de neumoquiste vertebral lumbar, describiendo su estudio, evolución y manejo.}

received

January 5, 2017

accepted

April 26, 2017

published online

June 30, 2017
DOI https://doi.org/

10.1055/s-0037-1603467.

ISSN 0716-4548.
Copyright $\odot 2017$ by Thieme Revinter

Publicações Ltda, Rio de Janeiro, Brazil

License terms

ㄷ) (i) $\ominus$ (\$) 
Abstract

\section{Keywords \\ - vertebral pneumatocyst \\ - lumbar spine \\ - case report}

The intraosseous pneumatocyst, is a rare condition in which there is gas-filled cystic lesion in the bone which is incidentally discovered on imaging studies and has been observed in relation to iliosacral joint, the humeral head, medial end of the clavicle, cervical spine, and thoracolumbar spine.

The computer tomography $(\mathrm{CT})$ is the most useful radiological tool for the diagnosis of the pneumatocyst and the pathognomonic finding is a cystic-like lesion with attenuation that ranges from 950 to 580 Hounsfield units, indicating gas, surrounded by a sclerotic rim.

Main differential diagnosis includes other possible causes of intraosseous gas such as infectious osteomyelitis, osteonecrosis, solitary bone cysts, and subchondral cysts. The natural progression of the intraosseous pneumatocyst is unclear. Some authors suggest that these lesions remained unchanged up to 4 years of follow up with $C T$, and even spontaneous resolution of the pneumatocyst have been reported, but others authors found progressive growth of the lesions in few weeks, months or years.

Surgical treatment could be indicated in symptomatic patients or with progressive growth of the lesion at CT follow up that could increase the potential risk of vertebral fracture.

We report a case of vertebral body intraosseous pneumoatocyst in lumbar spine describing the diagnosis process and management.

\section{Introducción}

Los neumoquistes intraoseos, son lesiones cavitadas de contenido gaseoso, las cuales generalmente son encontradas como hallazgos incidentales en exámenes imagenológicos. ${ }^{1,2}$ Es una patología escasamente descrita en la literatura, limitada a reportes de casos, entre los que se mencionan presentaciones a nivel sacroiliaco, columna cervical, y en menor medida, en la columna lumbar. Los neumoquistes intraóseos deben diferenciarse de otras causas de colecciones gaseosas intraóseas como osteomielitis, osteonecrosis o tumores óseos. ${ }^{3}$

Debido a la escasa evidencia respecto de esa lesión a nivel de la columna lumbar, es que se reporta un caso de neumoquiste vertebral lumbar además de una revisión de la literatura.

\section{Caso Clínico}

Paciente del sexo femenino de 49 años de edad, con antecedente de cáncer de tiroides operado y hernia de núcleo pulposo lumbar, diagnosticada hace 8 años, consulta por episodios de dolor lumbar sin irradiación, recurrentes, de tipo mecánico, sin síntomas neurológicos asociados. Refiere que en el año 2010 se le realiza bloqueo facetario lumbar con remisión de sintomatología durante 6 meses.

Al examen físico, presenta dolor lumbar al flexo-extensión y de movimientos rotacionales, sin déficit neurológico motor ni sensitivo de las extremidades inferiores, TEPE negativo, reflejos osteotendineos patelar y aquiliano conservados y simétricos. El examen de cadera y articulación sacroiliaca no presentaba hallazgos anormales.

Paciente trae tomografía computarizada de columna lumbar del año 2010, la que muestra una lesión ósea en cuerpo vertebral de $\mathrm{L} 5$ bien definida, de aspecto quístico.
Se solicita nueva tomografía computarizada de columna lumbar para evaluación de hallazgo radiológico, en la cual se confirma lesión ósea bien definida en aspecto posterior de cuerpo vertebral de L5 con densidad de gas (-985 UH) de medidas $16.8 \mathrm{~mm}, 11.9 \mathrm{~mm}$ y $12 \mathrm{~mm}$ en los ejes coronal, anteroposterior y transversal respectivamente. Se observa además discopatía degenerativa lumbosacra con pérdida de altura y erosión de plataformas vertebrales adyacentes, asociado a gas en el espacio discal (-Fig. 1).

Se solicita además una resonancia magnética de la columna lumbar en la que se informa la lesión ya descrita, hipointensa en secuencias $\mathrm{T} 1$ y $\mathrm{T} 2$, sin realce con el uso de Gadolinio endovenoso, además cambios Modic tipo 2 de plataformas vertebrales adyacentes (-Fig. 2).

Los hallazgos descritos sugieren un neumoquiste intraoseo a nivel de cuerpo vertebral de L5. Se decide mantener en observación la lesión vertebral y un manejo conservador de su patología degenerativa vertebral, catalogado como un síndrome de dolor lumbar triarticular, por lo que se realiza un bloqueo facetario lumbar más corticoides peridurales, esos últimos para el manejo del dolor discogénico asociado a la kinesioterapia. Paciente evoluciona en buenas condiciones, con disminución de dolor lumbar, sin limitación en las actividades de la vida diaria, y con indemnidad neurológica al examen físico, completando 12 sesiones de kinesioterapia con buena tolerancia. Se indica mantener pauta de ejercicios de estabilización y elongación a permanencia.

\section{Discusión}

El neumoquiste intraóseo fue descrito por primera vez en 1982 por Weinberg y Schneider como un ganglión intraóseo a nivel del hueso iliaco ${ }^{4}$, y en 1984 el grupo de Ramírez y col., 


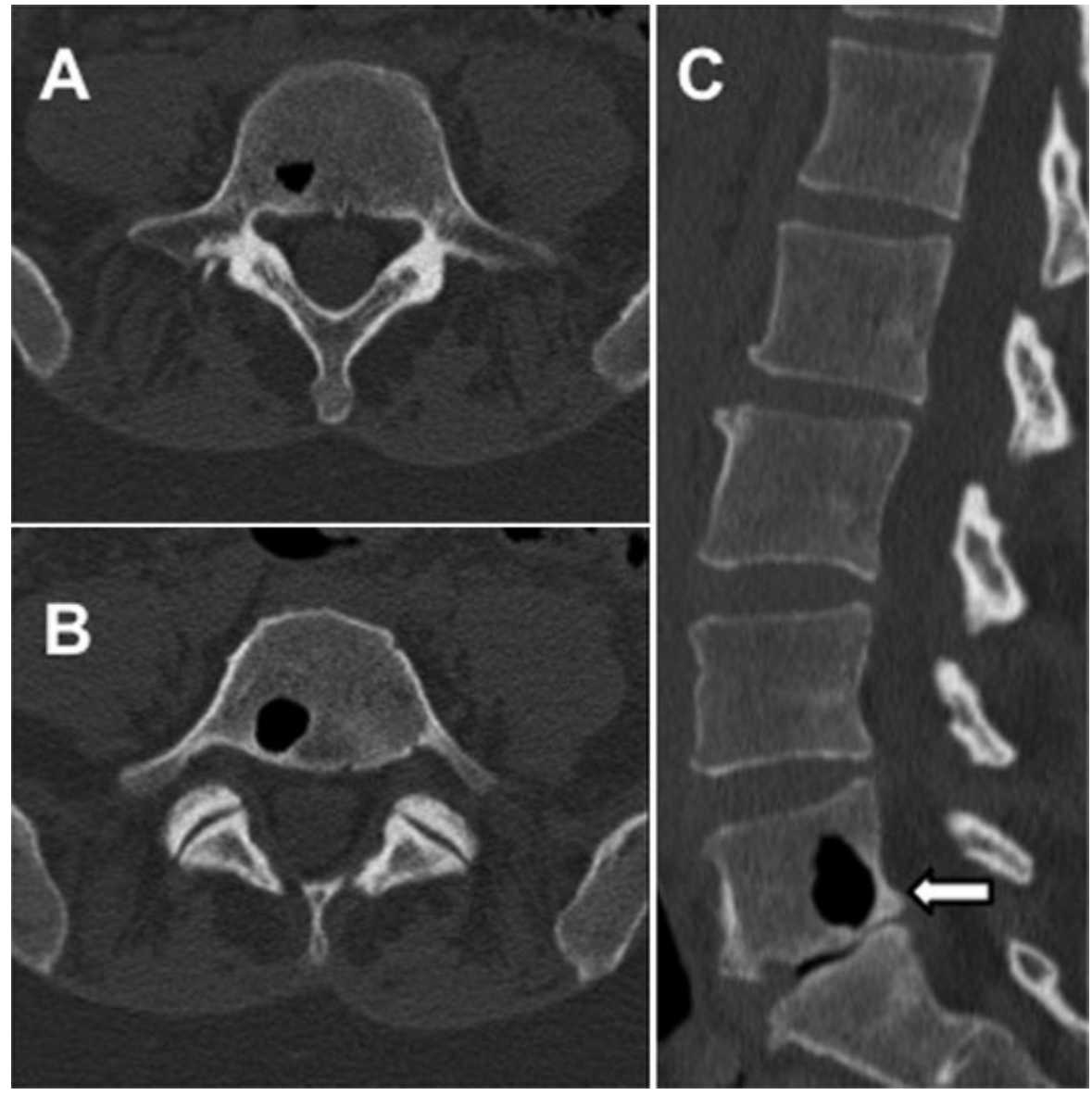

Fig. 1 Tomografía computarizada de columna lumbar sin contraste que muestra la lesión ósea bien definida en aspecto posterior de cuerpo vertebral $\mathrm{L} 5$ con densidad de gas (-985 UH). Se observa además discopatía degenerativa lumbosacra con pérdida de altura y erosión de plataformas vertebrales adyacentes, además de gas en el espacio discal.

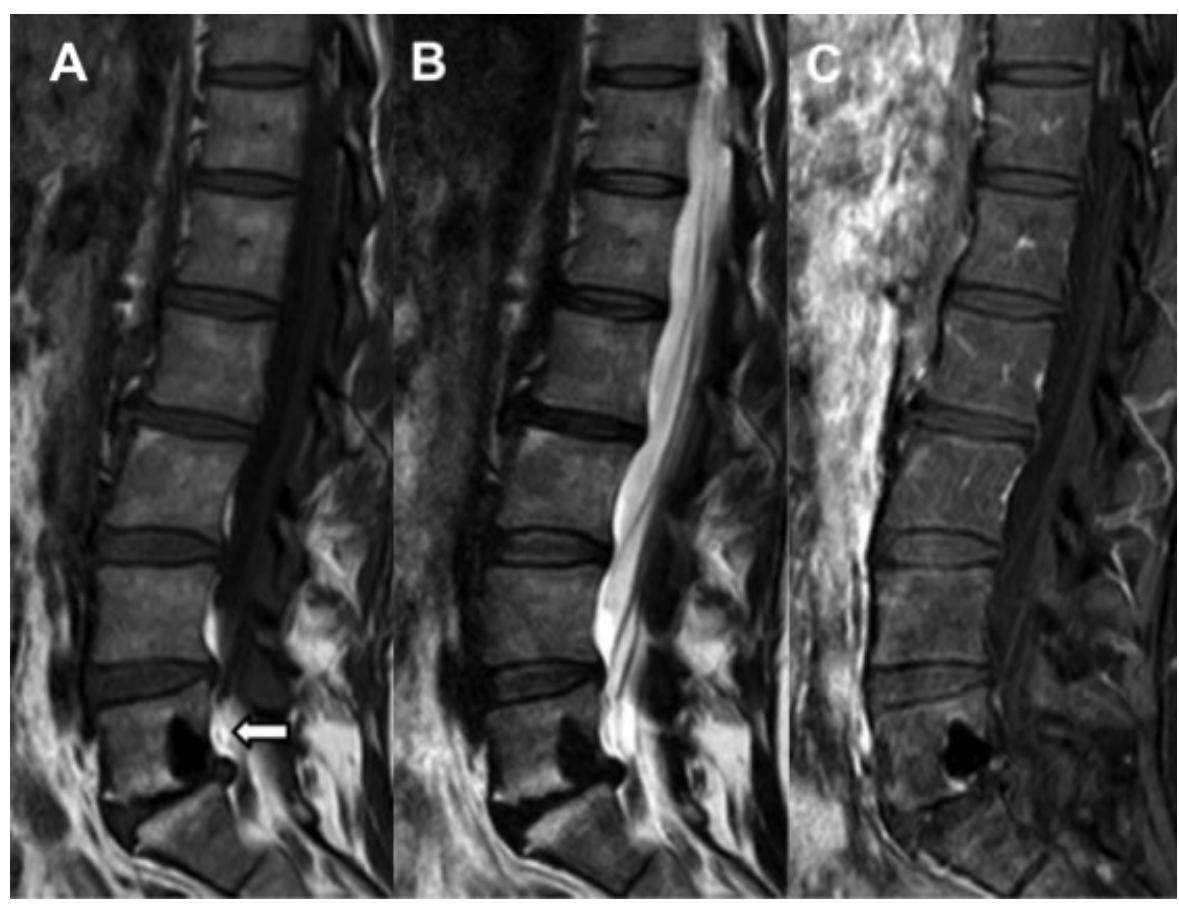

Fig. 2 Resonancia Magnética, secuencias T1 (A), T2 (B) y T1 con contraste endovenoso (C) muestra una lesión ósea bien definida en aspecto posterior de cuerpo vertebral L5, la cual es marcadamente hipointensa en secuencias T y T2, y no presenta realce con el uso de Gadolinio endovenoso. 
acuñaron el término neumoquiste en un paciente con una lesión sacroiliaca. Posteriormente a esos reportes, se han publicado diversos casos en la literatura de neumoquistes intraóseos, describiéndose en su mayoría como hallazgos incidentales a nivel sacroiliaco, pubis, sacro, clavícula, cabeza humeral, columna cervical, torácica y en menor medida columna lumbar. ${ }^{5}$

La prevalencia de esa lesión a nivel sacroiliaco es de un 10.3\% según el trabajo de Prassopoulos y col., y a nivel de columna cervical de un $9 \%$ según lo descrito por Arslan y col. ${ }^{1,6}$

La prevalencia a nivel lumbar es desconocida, ya que la evidencia se limita al reporte de casos aislados en la literatura.

En cuanto a la fisiopatología del neumoquiste vertebral, no se tiene claro el mecanismo por el cual ocurre esa lesión, sin embargo, se han postulado distintas teorías entre las que destacan la de Laufer y col., en la cual se describe que, debido a los cambios degenerativos articulares e intradiscales, asociado a cambios de presión, se produciría nitrógeno gaseoso el cual migraría a través del hueso subcondral hacia el cuerpo vertebral. Ese mecanismo fue denominado como "fenómeno de vacío". A partir de esa teoría, se podría explicar la mayor prevalencia de neumoquistes intraóseos en pacientes de mayor edad, asociado a cambios degenerativos vertebrales e intradiscales. ${ }^{7}$

Sin embargo, la teoría del fenómeno de vacío no lograría explicar la presencia de neumoquistes en pacientes sin lesiones degenerativas asociadas, como es el caso del grupo de pacientes jóvenes.

En el caso de neumoquistes intraóseos sin evidencia de lesiones degenerativas, se ha planteado que ellos se formarían a partir de quistes mucinosos o glangios intraóseos, en los cuales, de manera espontánea, ocurriría un reemplazo del contenido líquido por gas. Esa hipótesis estaría apoyada por análisis anatomopatológicos en los cuales se ha observado depósitos mucinosos en las muestras de tejido del neumoquiste intraóseo. ${ }^{4,8}$

A partir de los casos reportados en la literatura, se describe una mayor prevalencia en pacientes de sexo masculino en una proporción de 2.5 a $1 .^{5}$

En relación a la presentación clínica, esa lesión habitualmente ha sido reportada como asintomática, no presentando un cuadro característico, por lo que su diagnóstico corresponde a un hallazgo incidental en el estudio imagenológico de pacientes que se consultan por síntomas asociados a la patología degenerativa espinal concomitante. ${ }^{2,3}$

Kitagawa y col., plantean la presencia de dos grupos; por un lado, pacientes en un rango etario entre 49 y 69 años con evidencias de cambios degenerativos a nivel discal y vertebral, asociados al hallazgo del neumoquiste en el estudio imagenológico y en la contraparte, pacientes jóvenes, sin cambios degenerativos y con la presencia de un neumoquiste intravertebral de menor tamaño (2-5mm de diámetro). ${ }^{9}$

En cuanto a los estudios imagenológicos, como ya se mencionó previamente, el neumoquiste es un hallazgo incidental en la mayoría de los casos. En la radiografía simple, usualmente se presenta como una lesión radiolúcida de bordes escleróticos, sin embargo, en algunos reportes, se describen lesiones de aspecto osteolítico que podrían sugerir una lesión tumoral, por lo que el estudio se debe complementar con una tomografía computarizada (TC) y una resonancia magnética (RM). La TC es el examen de elección para realizar la confirmación diagnóstica y descartar diagnósticos diferenciales, y su patrón característico se describe como una lesión de aspecto quística con atenuación por gas intralesión con rangos entre -950 y -580 H. $^{5}$

Respecto a la RM, el neumoquiste vertebral es descrito como una lesión bien delimitada, con hipointensidad de señal en secuencias de $\mathrm{T}$ y $\mathrm{T} 2$, sin la presencia de realce con el uso de gadolinio. ${ }^{3,5}$

El diagnóstico diferencial debe incluir otras posibles causas de gas intraóseo como osteomielitis, osteonecrosis, quistes óseos o subcondrales, nódulos de Schmorl, o gas intraóseo debido a un trauma con herida penetrante asociada. ${ }^{5}$

Ante las manifestaciones clínicas y los patrones imagenológicos que no obedezcan a los elementos característicos descritos, o ante la duda diagnóstica, se sugiere la realización de una biopsia de la lesión, sin existir actualmente criterios definidos para ella. ${ }^{5}$

La progresión natural del neumoquiste intraoseo es desconocida. Distintos autores han descrito que en seguimientos radiológicos hasta 4 años, el tamaño de las lesiones se mantiene estable. ${ }^{10,11}$ Por otro lado, se han reportado casos en los cuales las lesiones han aumentado en tamaño en el seguimiento, entre los que destaca el reporte de Kitagawa y col., en el cual se observó un crecimiento progresivo del neumoquiste intraóseo durante 16 meses de seguimiento. ${ }^{9}$ Yamamoto y col., reportaron que en un seguimiento radiológico de 40 semanas, el neumoquiste progresó hacia una lesión quística con contenido líquido y posteriormente con tejido de granulación. ${ }^{10,12}$

En cuanto al manejo del neumoquiste intraóseo, en la mayoría de los casos reportados, se ha observado una evolución benigna, con manejo conservador de las lesiones, sin embargo, algunos autores como Nakagawa y col., recomiendan que en casos sintomáticos se debe realizar una excisión de la lesión con injerto óseo asociado, alcoholisación o inyección de cemento mediante punción bajo TC. ${ }^{13}$

Por otro lado Hoover y col., mencionan que frente a lesiones de gran tamaño con riesgo de fractura patológica, se debe plantear un manejo quirúrgico con corpectomía e injerto óseo. ${ }^{11}$

\section{Conclusión}

El neumoquiste intraóseo vertebral en su presentación lumbar es una lesión infrecuente, la cual se encuentra en un hallazgo incidental en estudios imagenológicos, y que posteriormente debe ser confirmada mediante TC y RM para descartar diagnósticos diferenciales. Actualmente, tanto la etiología como su historia natural es desconocida, formulándose distintas teorías basadas en el estudio de reportes de casos. Debido a todo eso, es que parece necesario seguir aportando con nuevos casos de neumoquistes intraóseos para poder alcanzar un conocimiento acabado de esa patología, y teniendo como objetivo un correcto diagnóstico y posteriormente una pauta para su manejo. 
70 Neumoquiste Vertebral en Columna Lumbar Fleiderman et al.

Fuente de Financiación

Este estudio no recibió financiación.

Declaración de Conflictos de Interés

Ninguno de los autores de este estudio presenta conflictos de interés.

\section{References}

1 Arslan G, Ceken K, Cubuk M, Ozkaynak C, Lüleci E. Vertebral pneumatocysts. Acta Radiol 2001;42(01):20-23

2 Coşar M, Eser O, Aslan A, et al. Vertebral body pneumatocyst in the cervical spine and review of the literature. Turk Neurosurg 2008; 18(02):197-199

3 Rensha H, Patel A, Boctor DS, Hakmi MA. 'Abnormal' cervical imaging?: Cervical pneumatocysts - A case report of a cervical spine pneumatocyst J Orthop 2014;12(01):83-85

4 Weinberg S, Schneider H. Case report 211. Skeletal Radiol 1982; 9(01):61-63

5 Oehler E, Valour F, Pascart T. Intraosseous pneumatocysts: a case based review. Clin Rheumatol 2013;32(01):129-134
6 Prassopoulos PK, Faflia CP, Goursoyiannis NC. Sacroiliac joint pneumatocysts: prevalence on CT examinations. Eur Radiol 1999;9(03):572

7 Laufer L, Schulman H, Hertzanu Y. Vertebral pneumatocyst. A case report. Spine 1996;21(03):389-391

8 Ramirez H Jr, Blatt ES, Cable HF, McComb BL, Zornoza J, Hibri NS. Intraosseous pneumatocysts of the ilium. Findings on radiographs and CT scans. Radiology 1984;150(02): 503-505

9 Kitagawa T, Fujiwara A, Tamai K, et al. Enlarging vertebral body pneumatocysts in the cervical spine. AJNR Am J Neuroradiol 2003;24(08):1707-1710

10 Sen D, Satija L, Saxena S, Rastogi V, Singh M. Intraosseous pneumatocyst of the cervical vertebra. Med J Armed Forces India 2015;71(04):380-383

11 Hoover JM, Wenger DE, Eckel LJ, Krauss WE. Cervical pneumatocyst. J Neurosurg Spine 2011;15(03):332-335

12 Yamamoto T, Yoshiya S, Kurosaka M, et al. Natural course of an intraosseous pneumatocyst of the cervical spine. AJR Am J Roentgenol 2002;179(03):667-669

13 Nakagawa T, Kawano H, Kubota T. Solitary bone cyst of the cervical spine-case report. Neurol Med Chir (Tokyo) 1994; 34(08):558-560 